\title{
786-0 Renal cancer cell line-derived exosomes promote 786-0 cell migration and invasion in vitro
}

\author{
GANG CHEN, YAO ZHANG and XIAOHOU WU \\ Department of Urology, The First Affiliated Hospital of Chongqing Medical University, \\ Yuzhong, Chongqing 400016, P.R. China
}

Received July 10, 2013; Accepted January 27, 2014

DOI: $10.3892 / \mathrm{ol} .2014 .1962$

\begin{abstract}
Emerging evidence indicates that cancer-derived exosomes contribute to angiogenesis, tumor immunology and invasion. However, whether cancer cell-derived exosomes regulate the migration and invasion of the cancer cell itself, and the underlying mechanisms are not well understood. In the present study, exosomes derived from the 786-0 human renal cancer cell line were isolated, purified and $100 \mu \mathrm{g} / \mathrm{ml}$ were co-cultured with 786-0 cells for $24 \mathrm{~h}$. The 786-0 cells were harvested for a cell invasion and migration assay. The expression of chemokine receptor type 4 (CXCR4) and matrix metalloproteinase-9 (MMP-9) in the 786-0 cells was examined by western blot analysis and revealed that the migration and invasion capabilities of the 786-0 cells were increased, however, the cell adhesion abilities were decreased as a result of the 24-h treatment with 786-0-derived exosomes. Furthermore, the expression levels of CXCR4 and MMP-9 in the 786-0 cells were increased. In conclusion, the 786-0 renal cancer cell line-derived exosomes increased migration and invasion, however, they decreased the adhesion ability of the 786- 0 cells. The exosomes may have increased the CXCR4 and MMP-9 expression levels in the 786-0 cells. These findings indicated that renal tumor-derived exosomes may contribute to renal cancer development and progression.
\end{abstract}

\section{Introduction}

Renal cell carcinoma (RCC) is one of the most common types of genitourinary cancer, accounting for $\sim 2-3 \%$ of all malignant tumors (1), and with an incidence of $\sim 209,000$ new cases and 102,000 mortalities per year worldwide. In Europe in 2008, 88,300 patients were diagnosed with RCC and 39,230 succumbed to the disease (2). With the development

Correspondence to: Professor Xiaohou $\mathrm{Wu}$, Department of Urology, The First Affiliated Hospital of Chongqing Medical University, 1 Youyi Road, Yuzhong, Chongqing 400016, P.R. China E-mail:wuxiaohou80@hotmail.com

Key words: renal cancer, cancer cell derived-exosomes, invasion, migration, chemokine receptor type 4 of diagnostic technology, an increasing number of patients with RCC are diagnosed at an early stage. However, a considerable number of patients with RCC present with metastasis at the time of diagnosis. Emerging evidence indicates that the development and progression of RCC are closely associated with the tumor microenvironment (3).

Exosomes are 30-100 nm-sized membrane vesicles, which are released into the tumor microenvironment by the majority of tumor cell types (4). Exosomes are currently recognized as important mediators of cell-to-cell communication, which transfer proteins, mRNAs and microRNAs to neighboring or distant cells to modulate immune function, angiogenesis, cell proliferation and tumor cell invasion (5-7). However, whether cancer cell-derived exosomes regulate the migration and invasion of the cancer cell itself and the underlying mechanisms have not yet been investigated.

Our previous study indicated that tumor cell-derived exosomes contributed to proliferation of the tumor cell (8). In the present study, exosomes were isolated from the 786-0 human renal carcinoma cell line and the effects of 786-0 cell-derived exosomes on the migration and invasion of 786-0 cells as well as the underlying mechanisms were investigated in vitro. Our findings may provide a novel insight into the mechanisms underlying the development and progression of RCC.

\section{Materials and methods}

Cell culture. The 786-0 human renal cancer cell line was purchased from the Institute of Cell Research, Chinese Academy of Sciences (Shanghai, China). The cells were cultured in RPMI-1640 medium (Gibco, Shanghai, China) supplemented with $10 \%$ (v/v) fetal bovine serum (FBS; Shijiqing Inc, Beijing, China) at $37^{\circ} \mathrm{C}$ in an atmosphere of $5 \%$ (v/v) $\mathrm{CO}_{2}$.

Isolation and purification of exosomes. The 786-0 cell-derived exosomes were isolated and purified as previously described (9). Briefly, cultured supernatants $(100 \mathrm{ml})$ were harvested and sequentially centrifuged $\left(4^{\circ} \mathrm{C}\right)$ at 300 , 800 and $10,000 \mathrm{x}$ g for 10,30 and $30 \mathrm{~min}$, respectively, to remove the pellet and cell debris. The clarified supernatant was then centrifuged at $1,000 \mathrm{x} \mathrm{g}$ for $30 \mathrm{~min}$ in a $100 \mathrm{kDa}$ MWCO Centrifugal Filter Device (Amicon Drive Systems, 
Inc., Pineville, NC, USA). The ultracentrifuge supernatant was underplayed with $30 \%$ sucrose/D2O density cushion, followed by ultracentrifugation at $100,000 \mathrm{x}$ g for $60 \mathrm{~min}$. The cushion was collected and diluted in phosphate-buffered saline (PBS). The exosomes were further concentrated by centrifuging at $1,000 \mathrm{x} \mathrm{g}$ in a $100 \mathrm{kDa} \mathrm{MWCO}$ for $30 \mathrm{~min}$. A membrane filter $(0.22 \mu \mathrm{m})$ was used and following sterilization the exosomes were stored at $-80^{\circ} \mathrm{C}$. The Bradford method was used to quantify the total protein concentration of exosomes.

Identification of exosomes. The morphological characteristics of the exosomes were identified under a transmission electron microscope (TEM; JEM-2010, JEOL, Ltd., Tokyo, Japan). The exosomes (20 $\mu \mathrm{l})$ were resuspended, loaded onto electron microscopy grids and stained with $2 \%$ phosphotungstic acid ( $\mathrm{pH}$ 6.8) for $1 \mathrm{~min}$. The samples were subjected to analysis under TEM at $80 \mathrm{kV}$. Western blotting was used to identify the molecular composition of G250, intercellular adhesion molecule-1 (ICAM-1) and $70 \mathrm{kDa}$ heat shock protein (Hsp70), which were purchased from Zhongshan Bio-tech Co., Ltd. (Guangdong, China).

Wound healing assay. The 786-0 cells were divided into three groups as follows: Exosomes (Exo), PBS (PBS) and exosomes depression (Exo-D) groups and pretreated with $100 \mu \mathrm{g} / \mathrm{ml}$ exosomes, $100 \mu \mathrm{l}$ PBS and $7 \mathrm{mmol} / 1$ Amiloride, 5-(N,N-Dimethyl)-, hydrochloride, respectively, for $24 \mathrm{~h}$. Each group was cultured in triplicate. The cells $\left(1 \times 10^{5}\right)$ were seeded in 12-well plates. After the cells formed a confluent monolayer, a $100 \mu \mathrm{l}$ tip was used to scratch the monolayer. The cultured medium was replaced with fresh complete medium and the cells were incubated at $37^{\circ} \mathrm{C}$ in an atmosphere of $5 \%(\mathrm{v} / \mathrm{v}) \mathrm{CO}_{2}$. The wound healing was analyzed under a microscope and images were captured $24 \mathrm{~h}$ following incubation.

Invasion assay. The cells were divided into three groups and pretreated in triplicate as described above. The cell invasion assay was performed using transwell chambers with $8 \mu \mathrm{m}$ pore-sized membranes coated with Matrigel (Collaborative Research, Inc., Bedford, MA, USA) and placed in 24-well plates. The $786-0$ cells $\left(1.5 \times 10^{4}\right)$ were harvested and loaded into the upper segment of the chambers. RPMI-1640 medium with 5\% FBS was added to the upper segment of the chamber and the lower chamber contained $10 \%$ FBS. Following incubation for $24 \mathrm{~h}$, the upper surfaces of the transwell chambers were wiped with cotton swabs, and the invading cells were fixed and stained with crystal violet solution. The invading cell numbers were counted in five randomly selected microscope fields.

Cell attachment assays. The 786-0 cells were divided into three groups and pretreated in triplicate as described above. The cells were harvested and seeded onto 6-well culture dishes in RPMI-1640 medium with 10\% FBS for $3 \mathrm{~h}$. Cell attachment assays were performed, non-adherent cells were removed and adherent cells were washed twice with PBS and fixed in 95\% alcohol. The cell numbers were counted in randomly selected high power fields under an inverted light microscope.
Western blot analysis. The 786-0 cells were pretreated as previously described for the wound healing assay. The cells were harvested and protein was extracted using a Protein Extraction kit according to the manufacturer's instructions (Active Motif, Carlsbad, CA, USA). The protein concentrations were determined by the Bradford protein assay. Total protein $(10 \mu \mathrm{g})$ was separated by SDS-PAGE and transferred onto a polyvinylidene difluoride membrane (Millipore, Billerica, MA, USA). The membranes were blocked with $5 \%$ skimmed milk in Trisbuffered saline containing $0.1 \%$ Tween-20 for $1 \mathrm{~h}$ and incubated with primary antibodies (1:200 dilution) against human chemokine receptor type 4 (CXCR4) and matrix metalloproteinase-9 (MMP-9) obtained from Tianjin Saier Biotechnology Co., Ltd. (Tianjin, China) overnight at $4^{\circ} \mathrm{C}$. The bound antibodies were detected with goat anti-rabbit IgG (Zhongshan Bio-tech Co., Ltd.) and visualized using enhanced chemiluminescence reagents (Millipore). The relative levels of each target protein to the control, $\beta$-actin, were determined by densitometric analysis using Image $\mathrm{J}$ software.

Statistical analysis. Data are expressed as the mean \pm standard deviation. The differences between the experimental groups were determined by Student's t-test using SPSS 19.0 (IBM, Armonk, NY, USA). P<0.05 was considered to indicate a statistically significant difference.

\section{Results}

Identification of exosomes derived from the 786-0 cells. The morphological characteristics of the exosomes derived from $786-0$ cells were identified by TEM. The 786- 0 cell-derived exosomes exhibited a typical characteristic of a cup-shaped or saucer-like structure and ranged from 30 to $100 \mathrm{~nm}$ in diameter (Fig. 1A). Western blot analysis revealed that 786-0 cell-derived exosomes expressed G250, ICAM-1 and Hsp70 (Fig. 1B). The Bradford protein assay found the protein concentration of prepared exosomes was $1,800.7 \pm 275.4 \mu \mathrm{g} / \mathrm{ml}$.

786-0 cell-derived exosomes enhance 786-0 cell motility. Amiloride, 5-(N,N-Dimethyl)-, hydrochloride was reported to depress the cell exosome secretion. In the wound healing assays, the 786-0 cells exhibited an enhanced migratory capacity to the wounded areas in the Exo group compared with the PBS and Exo-D groups (Fig. 2). The Matrigel invasion assays revealed that the 786-0 cells in the Exo group had a higher degree of motility compared with those in the blank control and the Exo-D groups. The invasion ability of cells in the Exo group $(87.5 \pm 7.8$ cells per field) was significantly higher compared with that of cells in the PBS $(57.6 \pm 5.4$ cells per field; $\mathrm{P}<0.05)$ and Exo-D $(39.3 \pm 11.7$ cells per field; $\mathrm{P}<0.05$ ) groups (Fig. 3).

786-0 cell-derived exosomes enhance 786-0 cell adhesion. In the cell attachment assays, cells in the Exo, PBS and Exo-D groups were inoculated in 6-well culture dishes. After the cells were incubated in 10\% FBS/ RPMI-1640 medium for $3 \mathrm{~h}$, significantly enhanced cell attachment was observed in the Exo-D group (71.5 \pm 7.5 cells per field) compared with attachment in the Exo $(42.5 \pm 6.5$ cells per field; $\mathrm{P}<0.05)$ and PBS $(51.5 \pm 8.5$ cells per field; $\mathrm{P}<0.05)$ groups (Fig. 4). These 

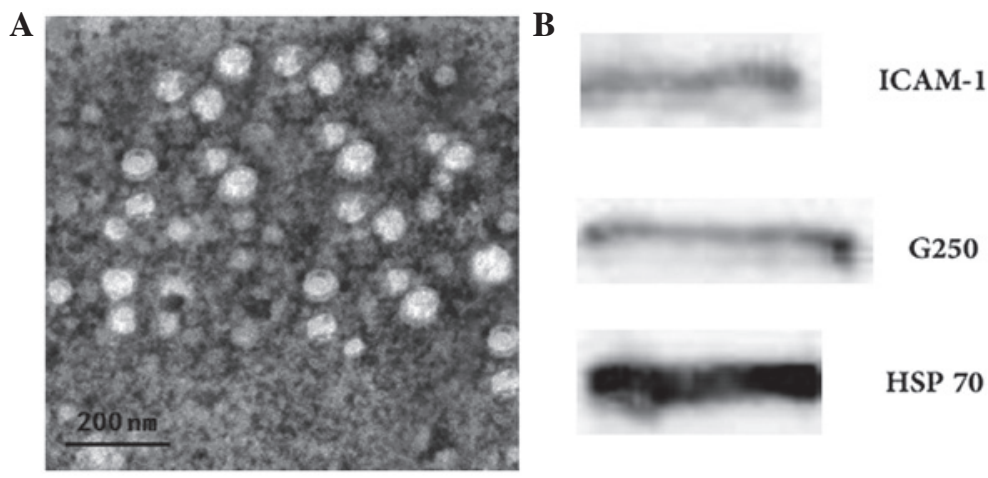

Figure 1. (A) Transmission electron microscopy of exosomes derived from 786-0 cells revealed typical characteristics of cup-shaped or saucer-like structures and 95\% ranged between 30 and $100 \mathrm{~nm}$ in diameter (magnification, x200). (B) Western blot analysis of 786-0 cell-derived exosomes revealed the expression of G250, ICAM-1 and HSP70. ICAM-1, intercellular adhesion molecule-1; Hsp70, $70 \mathrm{kDa}$ heat shock protein.

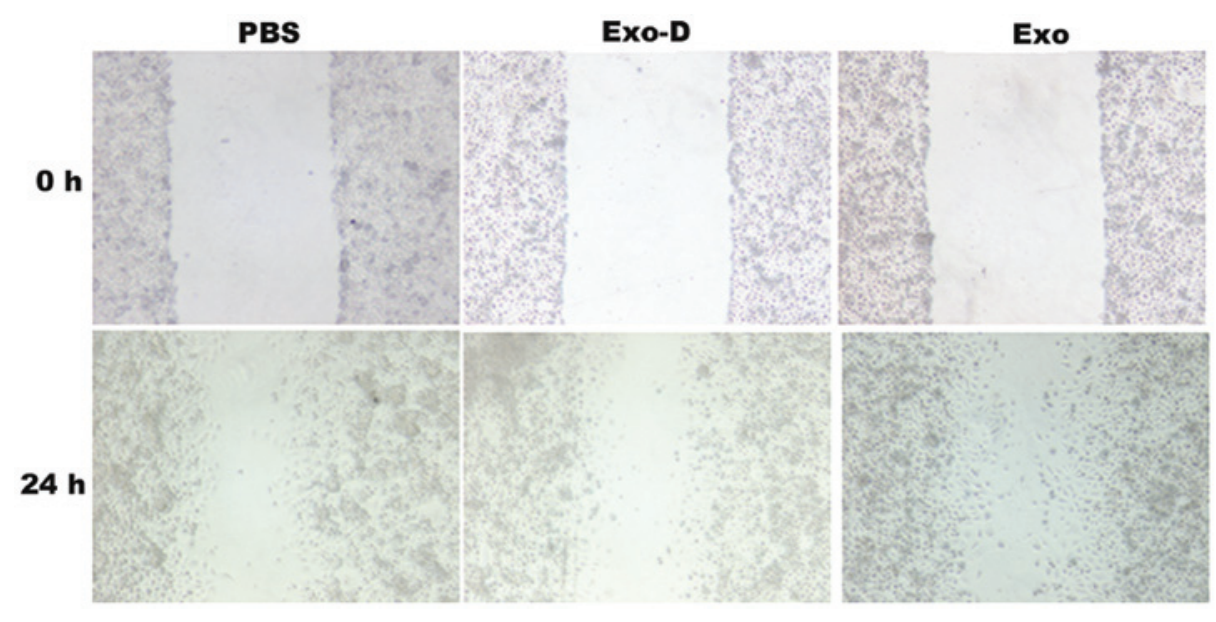

Figure 2. Migration capability of 786-0 cells was analyzed by the wound healing assay. Confluent monolayers of 786-0 cells were scratched and the repair was monitored microscopically after $24 \mathrm{~h}$ of pretreatment with Exo (100 $\mu \mathrm{g} / \mathrm{ml})$, PBS (7 mmol) and Amiloride, 5-(N,N-Dimethyl)-, hydrochloride (7 mmol; Exo-D) for $24 \mathrm{~h}$. The width of the wound area narrowed significantly in the Exo-treated group compared with the PBS and Exo-D groups $(\mathrm{P}<0.05)$. Exo, exosomes; PBS, phosphate-buffered saline; Exo-D, exosomes depression.

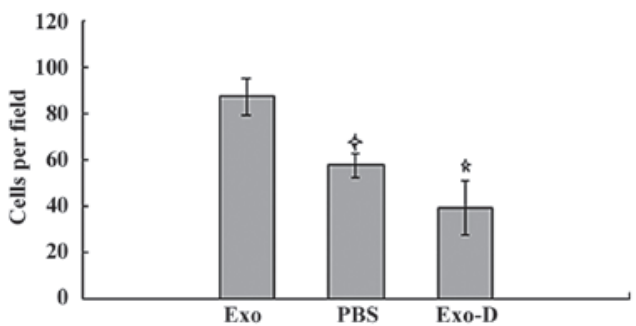

Figure 3. 786-0 cell-derived exosomes promote 786-0 cell invasion. 786-0 cells were pretreated with Exo $(100 \mu \mathrm{g} / \mathrm{ml})$, PBS $(7 \mathrm{mmol} / \mathrm{l})$ and Amiloride, 5-(N,N-Dimethyl)-, hydrochloride (7 mmol/1; Exo-D) for $24 \mathrm{~h} .786-0$ cells were seeded in the top chamber of the Matrigel. Following incubation, cells were assessed for invasion as described in the Materials and methods. The number of migrating cells in the Exo-group was significantly increased compared with the PBS and Exo-D groups $(\mathrm{P}<0.05)$. Exo, exosomes; PBS, phosphate-buffered saline; Exo-D, exosomes depression. ${ }^{*} \mathrm{P}<0.05$ compared with the Exo group.

findings indicated that 786-0 cell-derived exosomes may decrease the adhesion ability of 786-0 cells.

786-0 cell-derived exosomes increase 786-0 cell migration and invasion via the CXCR4 and MMP-9 signaling pathways.

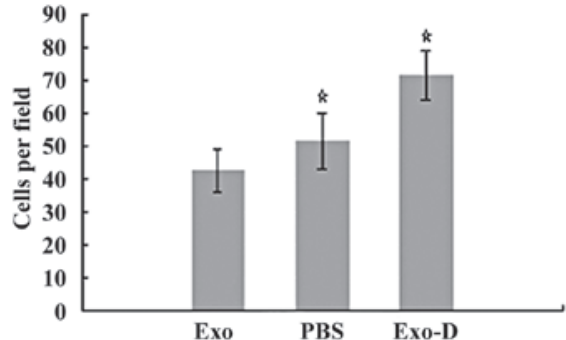

Figure 4. 786-0 cell-derived exosomes decrease 786-0 cell attachment 786-0 cells were pretreated with Exo $(100 \mu \mathrm{g} / \mathrm{ml})$, PBS $(7 \mathrm{mmol} / \mathrm{l})$ and Amiloride, 5-(N,N-Dimethyl)-, hydrochloride (7 mmol/l; Exo-D) for $24 \mathrm{~h}$. The cell attachment assays were processed as described in the Materials and methods. Cells in the Exo-D group showed significant enhanced cell attachment to the culture dishes compared with the cells in the Exo and PBS-treated groups $(\mathrm{P}<0.05)$. Exo, exosomes; PBS, phosphate-buffered saline; Exo-D, exosomes depression. " $\mathrm{P}<0.05$ compared with Exo-D group.

To determine whether exosomes increase 786-0 cell migration and invasion via the CXCR4 and MMP-9 signaling pathways, the protein expression of CXCR4 and MMP-9 was examined by western blott analysis. The protein expression levels of CXCR4 and MMP-9 were identified to be significantly enhanced in 


\section{CXCR4 MMP-9}

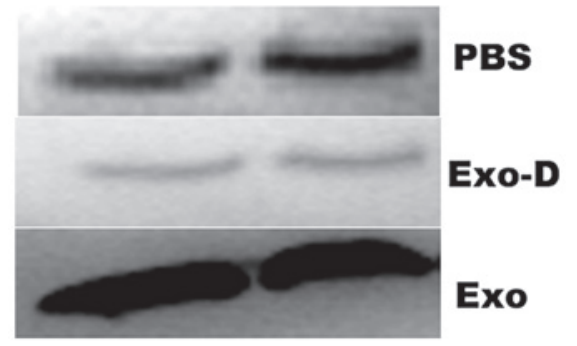

Figure 5.786-0 cell-derived exosomes increase CXCR4 and MMP-9 expression. 786-0 cells were pretreated with Exo $(100 \mu \mathrm{g} / \mathrm{ml})$, PBS $(7 \mathrm{mmol} / \mathrm{l})$ and Amiloride, 5-(N,N-Dimethyl)-, hydrochloride (7 mmol/l; Exo-D) for $24 \mathrm{~h}$. Total protein was extracted for western blot analysis. CXCR4 and MMP-9 expression were significantly increased in the Exo-treated group compared with the Exo-D and PBS-treated groups $(\mathrm{P}<0.05)$. Exo, exosomes; PBS, phosphate-buffered saline; Exo-D, exosomes depression; CXCR4, chemokine receptor type 4; MMP-9, matrix metalloproteinase-9.

the Exo group compared with the PBS group, however, were significantly reduced in the Exo-D group (Exo-D) compared with the PBS group (Fig. 5).

\section{Discussion}

Surgery is the primary curative therapy for patients with local RCC. The treatment for metastatic RCC (mRCC) has been a focus of investigation, however, remains unclear. Analysis of interleukin-2, vascular endothelial growth factor (VEGF) and mammalian target of rapamycin inhibitors have provided improvements in the clinical outcomes of mRCC (10). However, the prognosis for advanced $\mathrm{mRCC}$ patients remains poor, with a five-year survival rate of $<10 \%$ (11). Therefore, further investigations into the underlying mechanisms of mRCC are required.

Tumor-derived exosomes have a bimodal role in cancer progression. First, exosomes regulate the local and systemic environment and contribute to cancer growth and dissemination in vivo. Second, exosomes may be important in eliciting the antitumor responses of the immune system (12). Our previous study demonstrated that tumor-derived exosomes enhanced tumor cell proliferation and inhibited Jurkat T-cell proliferation in vitro (8). However, whether tumor-derived exosomes enhanced migration and invasion of the cancer cell itself in vitro, was not clarified. In the present study, purified exosomes derived from the 786-0 human RCC cell line exhibited typical characteristics, including cup-shaped or saucer-like structures, and ranged between 30 and $100 \mathrm{~nm}$ in diameter. Protein analysis revealed that the 786-0 cell-derived exosomes expressed G250, ICAM-1 and Hsp70. In addition, treatment with $100 \mu \mathrm{g} / \mathrm{ml}$ exosomes enhanced cell migration and invasion, but decreased cell attachment in vitro. However, the underlying mechanisms by which exosomes enhanced the migration and invasion of 786-0 cells remains unclear.

The invasion of tumor cells is a complex, multistage process, which includes the degradation, by proteases, of the extracellular matrix (ECM) and the basement membrane surrounding the primary tumor, as well as enhancing cell migration and invasion $(13,14)$. Numerous molecules have been associated with cancer metastasis, including MMPs, VEGFs, tumor necrosis factor, platelet-derived growth factor, transforming growth factor- $\beta$ and Twist-related protein 1 (15). Chemokines and their receptors have been found to contribute to cancer metastasis, particularly stromal cell-derived factor- $1 \alpha$ and its receptor CXCR4 $(16,17)$. The CXCR4 chemokine receptor is highly expressed in various types of tumors, including breast, gastric, ovarian, pancreatic, prostate and renal cancers and acute myeloid leukemia (18). Tumors expressing CXCR4 acquire properties that enable them to invade tissue barriers, migrate to secondary organs and form metastases. In patients with RCC, CXCR4 expression has been correlated with a poor overall survival and the expression of CXCR4 and CXCR7 has been considered as a marker, with $\sim 80 \%$ accuracy, for predicting $\mathrm{RCC}$ metastasis $(19,20)$. As CXCR4 has been associated with the metastasis of renal cancers, CXCR4 expression may be a potential molecular marker for the increased cell invasion and migration abilities, which are induced by tumor-derived exosomes.

MMP-9 is a downstream signaling molecule of CXCR4 and is critical for the migration and invasion of cancer cells (21). MMP-9 belongs to a large family of MMPs, which are responsible for degrading a wide range of ECM components (22). Additionally, MMP-9 is closely associated with tumor invasion and metastasis in a variety of human tumors, including lung adenocarcinoma, hepatocellular carcinoma, and prostate and breast cancers (23-26); furthermore, it was expressed in the serum and urine of patients with RCC (27). Downregulation of forkhead box protein M1 reduced the expression and activity of MMP-2, -9 and VEGF, resulting in the inhibition of migration, invasion and angiogenesis of renal cancer cell lines (28). In the present study, 786-0 cells treated with $100 \mu \mathrm{g} / \mathrm{ml} 786-0$ cell-derived exosomes showed high migration and invasion capabilities and an increased expression of CXCR4 compared with the PBS-treated group. These findings indicated that the CXCR4 and MMP-9 signaling pathways may be involved in the enhanced migration and invasion capability of 786-0 cells, which was induced by the 786-0 cell-derived exosomes.

In conclusion, these data demonstrated that exosomes, which were derived from the 786-0 human RCC cell lines induced the high migration and invasion capabilities of 786- 0 cells in vitro. The tumor-derived exosomes induced the tumor cell to highly express CXCR4 and MMP-9. To the best of our knowledge, this is the first study to report that 786-0 cell-derived exosomes enhanced migration and invasion of 786-0 cancer cells. However, further investigations are required to elucidate whether renal tumor-derived exosomes degrade the ECM and promote renal tumor invasion in vivo. Our findings may provide novel insights into the tumor progression of RCC.

\section{Acknowledgements}

The present study was supported by a research grant from the Foundation of National Natural Science Foundation of China (grant no. 81272572). The authors would like to thank Professors Luo Chun-Li and Yin Zhi-Kang. 


\section{References}

1. Ferlay J, Parkin DM and Steliarova-Foucher E: Estimates of cancer incidence and mortality in Europe in 2008. Eur J Cancer 46: 765-781, 2010.

2. Jemal A, Siegel R, Xu J and Ward E: Cancer statistics. CA Cancer J Clin 60: 277-300, 2010.

3. Milella $M$ and Felici A: Biology of metastatic renal cell carcinoma. J Cancer 2: 369-373, 2011.

4. Théry C, Ostrowski M and Segura E: Membrane vesicles as conveyors of immune responses. Nat Rev Immunol 9: 581-593, 2009.

5. Balaj L, Lessard R, Dai L, Cho YJ, Pomeroy SL, Breakefield XO and Skog J: Tumour microvesicles contain retrotransposon elements and amplified oncogene sequences. Nat Commun 2 : 180,2011

6. Valadi H, Ekström K, Bossios A, Sjöstrand M, Lee JJ and Lötvall JO: Exosome-mediated transfer of mRNAs and microRNAs is a novel mechanism of genetic exchange between cells. Nat Cell Biol 9: 654-659, 2007.

7. Kahlert C and Kalluri R: Exosomes in tumor microenvironment influence cancer progression and metastasis. J Mol Med (Berl) 91 431-437, 2013.

8. Yang L, Wu XH, Wang D, Luo CL and Chen LX: Bladder cancer cell-derived exosomes inhibit tumor cell apoptosis and induce cell proliferation in vitro. Mol Med Rep 8: 1272-1278, 2013.

9. Zhang Y, Luo CL, He BC, Zhang JM, Cheng G and Wu XH: Exosomes derived from IL-12-anchored renal cancer cells increase induction of specific antitumor response in vitro: A novel vaccine for renal cell carcinoma. Int J Oncol 36: 133-140, 2010.

10. Weiss C, Schulze B, Ottinger A and Rödel C: To combine or not combine: the role of radiotherapy and targeted agents in the treatment for renal cell carcinoma. World J Urol: May 8, 2013 (Epub ahead of print)

11. Coppin C, Kollmannsberger C, Le L, Porzsolt F and Wilt TJ Targeted therapy for advanced renal cell cancer (RCC): a Cochrane systematic review of published randomised trials. BJU Int 108: 1556-1563, 2011.

12. Yang $\mathrm{C}$ and Robbins PD: The roles of tumor-derived exosomes in cancer pathogenesis. Clin Dev Immunol 2011: 842849, 2011.

13. Samara GJ, Lawrence DM, Chiarelli CJ, Valentino MD, Lyubsky S, Zucker S and Vaday GG: CXCR4-mediated adhesion and MMP-9 secretion in head and neck squamous cell carcinoma. Cancer Lett 214: 231-241, 2004.

14. Yu T, Wu Y, Helman JI, Wen Y, Wang C and Li L: CXCR4 promotes oral squamous cell carcinoma migration and invasion through inducing expression of MMP-9 and MMP-13 via the ERK signaling pathway. Mol Cancer Res 9: 161-172, 2011.

15. Park B, Sung B, Yadav VR, Cho SG, Liu M and Aggarwal BB Acetyl-11-keto- $\beta$-boswellic acid suppresses invasion of pancreatic cancer cells through the downregulation of CXCR4 chemokine receptor expression. Int J Cancer 129: 23-33, 2011.
16. Raman D, Baugher PJ, Thu YM and Richmond A: Role of chemokines in tumor growth. Cancer Lett 256: 137-165, 2007.

17. Orimo A, Gupta PB, Sgroi DC, Arenzana-Seisdedos F, Delaunay T, Naeem R, Carey VJ, Richardson AL and Weinberg RA: Stromal fibroblasts present in invasive human breast carcinomas promote tumor growth and angiogenesis through elevated SDF-1/CXCL12 secretion. Cell 121: 335-348, 2005.

18. Manu KA, Shanmugam MK, Rajendran P, Li F, Ramachandran L, Hay HS, Kannaiyan R, Swamy SN, Vali S, Kapoor S, et al: Plumbagin inhibits invasion and migration of breast and gastric cancer cells by downregulating the expression of chemokine receptor CXCR4. Mol Cancer 10: 107, 2011.

19. Wang L, Chen W, Gao L, Yang Q, Liu B, Wu Z, Wang Y and Sun Y: High expression of CXCR4, CXCR7 and SDF-1 predicts poor survival in renal cell carcinoma. World J Surg Oncol 10: 212, 2012.

20. Gahan JC, Gosalbez M, Yates T, Young EE, Escudero DO, Chi A, Garcia-Roig M, Satyanarayana R, Soloway MS, Bird VG and Lokeshwar VB: Chemokine and chemokine receptor expression in kidney tumors: molecular profiling of histological subtypes and association with metastasis. J Urol 187: 827-833, 2012.

21. Zhao M, Gao Y, Wang L, Liu S, Han B, Ma L, Ling Y, Mao S and Wang X: Overexpression of integrin-linked kinase promotes lung cancer cell migration and invasion via NF- $\mathrm{kB}$-mediated upregulation of matrix metalloproteinase-9. Int J Med Sci 10: 995-1002, 2013.

22. Jia W, Gao XJ, Zhang ZD, Yang ZX and Zhang G: S100A4 silencing suppresses proliferation, angiogenesis and invasion of thyroid cancer cells through downregulation of MMP-9 and VEGF. Eur Rev Med Pharmacol Sci 17: 1495-1508, 2013.

23. Shiau MY, Fan LC, Yang SC, Tsao CH, Lee H, Cheng YW, Lai LC and Chang YH: Human papillomavirus up-regulates MMP-2 and MMP-9 expression and activity by inducing interleukin-8 in lung adenocarcinomas. PLoS One 8: e54423, 2013.

24. Dai ZJ, Wang BF, Lu WF, Wang ZD, Ma XB, Min WL, Kang HF, Wang XJ and Wu WY: Total flavonoids of Scutellaria barbata inhibit invasion of hepatocarcinoma via MMP/TIMP in vitro. Molecules 18: 934-950, 2013.

25. Wang X, Lee SO, Xia S, Jiang Q, Luo J, Li L, Yeh S and Chang C: Endothelial cells enhance prostate cancer metastasis via IL- $6 \rightarrow$ androgen receptor $\rightarrow$ TGF- $\beta \rightarrow$ MMP-9 signals. Mol Cancer Ther 12: 1026-1037, 2013.

26. Leifler KS, Svensson S, Abrahamsson A, Bendrik C, Robertson J, Gauldie J, Olsson AK and Dabrosin C: Inflammation induced by MMP-9 enhances tumor regression of experimental breast cancer. J Immunol 190: 4420-4430, 2013.

27. DI Carlo A: Evaluation of neutrophil gelatinase-associated lipocalin (NGAL), matrix metalloproteinase-9 (MMP-9) and their complex MMP-9/NGAL in sera and urine of patients with kidney tumors. Oncol Lett 5: 1677-1681, 2013.

28. Xue YJ, Xiao RH, Long DZ, Zou XF, Wang XN, Zhang GX, Yuan YH, Wu GQ, Yang J, Wu YT, et al: Overexpression of FoxM1 is associated with tumor progression in patients with clear cell renal cell carcinoma. J Trans1 Med 10: 200, 2012. 